\title{
Close pairs of quasars with different redshifts: New observations and results
}

\author{
D. Sluse ${ }^{1,2}$, J. Surdej ${ }^{1, \star}$, J.-F. Claeskens ${ }^{1, \star \star}$, Y. De Rop ${ }^{1}$, D. W. Lee ${ }^{1}$, A. Iovino ${ }^{3}$, and M. R. S. Hawkins ${ }^{4}$ \\ ${ }^{1}$ Institut d'Astrophysique et de Géophysique, Université de Liège, Allée du 6 Août, 17, B5C, 4000 Sart Tilman, Belgium \\ 2 European Southern Observatory, Alonso de Cordova 3107, Santiago 19, Chile \\ 3 Osservatorio Astronomico di Brera, Via Brera 28, 20121 Milano, Italy \\ ${ }^{4}$ Royal Observatory, Blackford Hill, Edinburgh EH9 3HJ, Scotland, UK
}

Received 15 November 2001 / Accepted 15 October 2002

\begin{abstract}
Burbidge et al. (1997) argue that the observed number of quasar pairs with small angular separations and different redshifts (typically $\Delta \theta \leq 5^{\prime \prime}$ and $\Delta z>0.1$ ) is not compatible with a random distribution of quasars over the sky. After a brief review of all known quasar pairs with different redshifts, we show by means of very simple calculations that the probability of finding the three accepted pairs accidentally is of the order of $10 \%$. We conclude that, under realistic hypotheses, the observed number of quasar pairs with different redshifts is not unlikely. We also present arguments showing that gravitational lensing biases are probably not strong enough to significantly increase the expected number of quasar pairs. The failure to detect with HST a secondary lensed image of the background quasar near the foreground one in these three pairs supports this view.
\end{abstract}

Key words. quasars: general - gravitational lensing

\section{Introduction}

In 1973, Wampler et al. discovered the pair of quasars $\mathrm{Q} 1548+114 \mathrm{~A}$ and $\mathrm{B}$, with redshifts $z_{\mathrm{A}}=0.44$ and $z_{\mathrm{B}}=1.9$, separated on the sky by only $4.8^{\prime \prime}$. This discovery was reported to be very unusual. Indeed, using simple statistical arguments, Wampler et al. have calculated that there was a chance of $1 \%$ to discover such a configuration by accident. In 1997, three additional pairs had been proposed. On this basis, Burbidge, Hoyle and Schneider (hereafter BHS97) evaluated a probability of only $0.35 \%$ to discover the four close quasar pairs proposed at that time. Such a small probability value could suggest that quasars are not randomly distributed over the sky and that close quasar pairs with different redshifts might represent physically associated objects. In other words, claims have been made that there could exist a non cosmological component to the redshift of quasars. Another possible interpretation of this result, as suggested by Schneider (in BHS97) and by Wampler (1997), is to consider that the statistical calculations are biased by the gravitational lensing amplification effect. Indeed, if we consider that the foreground quasar has a non negligible mass and that it could be a member of a massive cluster, it could induce a significant amplification on the light received from the background object. Due to this, a larger population of

Send offprint requests to: D. Sluse, e-mail: dsluse@eso.org

* Directeur de recherches du F.N.R.S. (Belgium).

$\star \star$ Chargé de recherches du F.N.R.S. (Belgium). intrinsically fainter quasars is seen and considered in the calculations as belonging to a brighter and less numerous one.

In order to test this hypothesis, we have used the large dynamical range and high angular resolution of the WFPC 2 onboard the Hubble Space Telescope (HST) to look among the different quasar pairs for the presence of a secondary lensed image of the background source near the foreground quasar. The presence of such images should help in constraining the mass of the lens and would confirm the assumption of strong amplification proposed in BHS97. As it could be foreseen from the large observed angular separation of typically 4 " between the quasars in the pairs, no secondary lensed image has been found; indeed typical angular separations produced by a lensing galaxy between multiple QSO images are expected to be in the $1^{\prime \prime}-2^{\prime \prime}$ range (see Claeskens \& Surdej 2002 for a list of the known gravitationally lensed systems). Iovino \& Shaver (1986) and Claeskens et al. (2000, 2001; hereafter Papers I and II) have used this absence of a secondary lensed image to set an upper limit to the mass of the foreground quasar and to show that for any realistic lens model, the foreground quasar and its cluster environment, whenever visible, only induce a small amplification on the background QSO images.

In the present article, we first review in Sect. 2 HST observations of the known close quasar pairs and their implications on the mass of the foreground quasar and on the amplification of the background one. After a few remarks on the content of the Véron \& Véron (2000; hereafter VV2000) 
catalogue in Sect. 3, we expose and discuss our estimates on the probability of finding the three accepted quasar pairs in that catalogue (Sect. 4).

\section{Observational data}

The VV2000 catalogue contains three close quasar pairs with different redshifts: Q1548+114 A\&B, Q1009-0252 A, B \& C and Q1148+0055 A \& B. Their characteristics are summarized in Table 1. The triple system Q1009-0252 is found to be composed of a foreground quasar at redshift 1.627 and of a background quasar $(z=2.746)$ gravitationally lensed by an intervening galaxy at $z=0.87$ (see Sect. 2.2). Since Q1009-0252 A $\& \mathrm{~B}$ has been identified as two lensed images of the same object, it has not been counted as a pair and we are left with the pair Q1009-0252 A \& C. AO 0235+164, considered as a 2.5" quasar pair by BHS97, has been excluded in the present study. Indeed there is no quasar in this system which is composed of a bright highly variable blazard at redshift 0.94 and of an AGN with $M_{\mathrm{B}}=-21.3$ (see e.g. Spinrad 1975; Urry et al. 2000). A spiral galaxy constitutes the 3rd component of this system (Burbidge et al. 1996).

The high angular resolution of the HST images obtained for these objects between January and April 1999 with the WFPC2 planetary camera (see Papers I and II and Fig. 1) have enabled us to put more stringent observational constraints on the possible presence of a secondary lensed image in the vicinity of the foreground lens quasar and, by the way, to predict the amplification of the background object together with an upper mass estimate for the foreground quasar.

\section{1. $Q 1548+114$ and $Q 1148+0055$}

The most favourable case for the formation of a secondary lensed QSO image is Q1548+114; this system has been studied in Paper I altogether with Q1148+0055. In that paper, Q1548+114 A has been modeled with a point mass and its host galaxy with a truncated Spherical Isothermal Sphere (SIS). All the galaxies located within $45^{\prime \prime}$ from Q1548+114 A have also been modeled by means of truncated SISs and, finally, a uniform sheet of matter has been used to represent the foreground galaxy cluster. We have then been able to predict for different values of the quasar mass, the expected magnitude difference between the two putative lensed QSO images as a function of their angular separation. Combined with the constraints on the non observability of a secondary image (see Paper I for details), we have deduced the maximum quasar mass for given $\mathrm{M} / \mathrm{L}$ ratios and densities of the sheet of matter. For Q1548+114 A, we have found an upper mass limit of $4.5 \times 10^{11} M_{\odot}$, and $6.5 \times 10^{11} M_{\odot}$ for the case of Q1148+0055 A. For Q1548+114, the absolute amplification of the primary image has been evaluated in two realistic situations with a foreground quasar mass fixed to $10^{11} M_{\odot}$. The first one corresponds to galaxies with $M / L$ ratios of 30 and a density sheet of dark matter of $0.1 \mathrm{~g} / \mathrm{cm}^{2}$ $(\kappa=0.2)$, while the second one is for $M / L=100$ and $\kappa=0$. We found for these two cases, an amplification of 1.67 and 1.72, respectively. Finally, a maximum value of 1.06 is obtained for the absolute amplification of Q1148+0055 B, if $M / L=100$.
Table 1. QSO identifier, redshift, magnitude (based on HST data) and angular separation of the known quasar pairs. The $B$ magnitude of Q1548+114 B has been derived from the $R$ magnitude (see Sect. 3.2).

\begin{tabular}{l|c|c|c|c|c}
\hline \hline QSO Id. & $z_{\mathrm{A}}$ & $z_{\mathrm{B} / \mathrm{C}}$ & $B_{\mathrm{A}}$ & $B_{\mathrm{B} / \mathrm{C}}$ & Sep \\
\hline Q1548+114A-B & 0.436 & 1.901 & 17.47 & 19.16 & $4.8^{\prime \prime}$ \\
Q1009-0252A-B & 2.746 & 2.746 & 18.2 & 21.2 & $1.55^{\prime \prime}$ \\
Q1009-0252A-C & 2.746 & 1.627 & 18.2 & 19.3 & $4.6^{\prime \prime}$ \\
Q1148+0055A-B & 1.879 & 1.409 & 18.48 & 21.13 & $3.9^{\prime \prime}$ \\
\hline
\end{tabular}

\section{2. $Q 1009-0252$}

As we may see from Fig. 1, Q1009-0252 is a particular case for which the background component is a doubly imaged QSO. It is mainly produced by the nearby lens galaxy $\mathrm{G}$ with a likely redshift $z=0.87$, very well seen on the F814W HST CCD frames, and not by the quasar Q1009-0252 C at a redshift $z=1.627$ (see Paper II for a complete study of this system and the estimate of the photometric redshift of the lens). The lens has been modeled with a SIS model+shear. The fit of the various model parameters on the observed configuration has enabled us to derive an absolute amplification of 2.86 for Q1009-0252 A. Nevertheless, Claeskens et al. (2001) found that the lensing model is close to the SIS with a very small shear value (0.017) which suggests that $95 \%$ of the amplification is due to the lens galaxy.

\subsection{The gravitational lensing amplification bias}

Typical values for the background amplification are difficult to estimate because the latter is not only a function of the angular separation between the foreground mass and the background source, but it also depends on the redshifts of the different components and on the lens model. Nevertheless, for the various observed pairs, we have shown that the foreground quasar and its environment produce an amplification of $0.5 \mathrm{mag}$, at maximum. Such a value, introduced in the model of Schneider in BHS97, implies an increase in the number of expected pairs by less than a factor 2 . According to the values of the amplification derived for the observed pairs, this factor is probably overestimated, but we shall consider this case as an extreme one for the possible gravitational lensing amplification bias.

\section{The Véron \& Véron catalogue}

We have searched the VV2000 catalogue for close $\left(\leq 5^{\prime \prime}\right)$ quasar pairs with different redshifts. This catalogue lists the position, magnitude and redshift of the whole population of quasars already discovered and identified (i.e. 13213 objects). Since it is intrinsically heterogeneous, it is not designed for statistical studies. Nevertheless, we may reasonably assume that a significant number of quasars in VV2000 have been observed or re-observed with a high angular resolution, so that nearby companions, if present at all, would have been discovered and reported. Besides published results, we searched the HST database and we made an inquiry among regular quasar observers and gravitational lens investigators to confirm this assumption. We found evidences of high angular resolution 

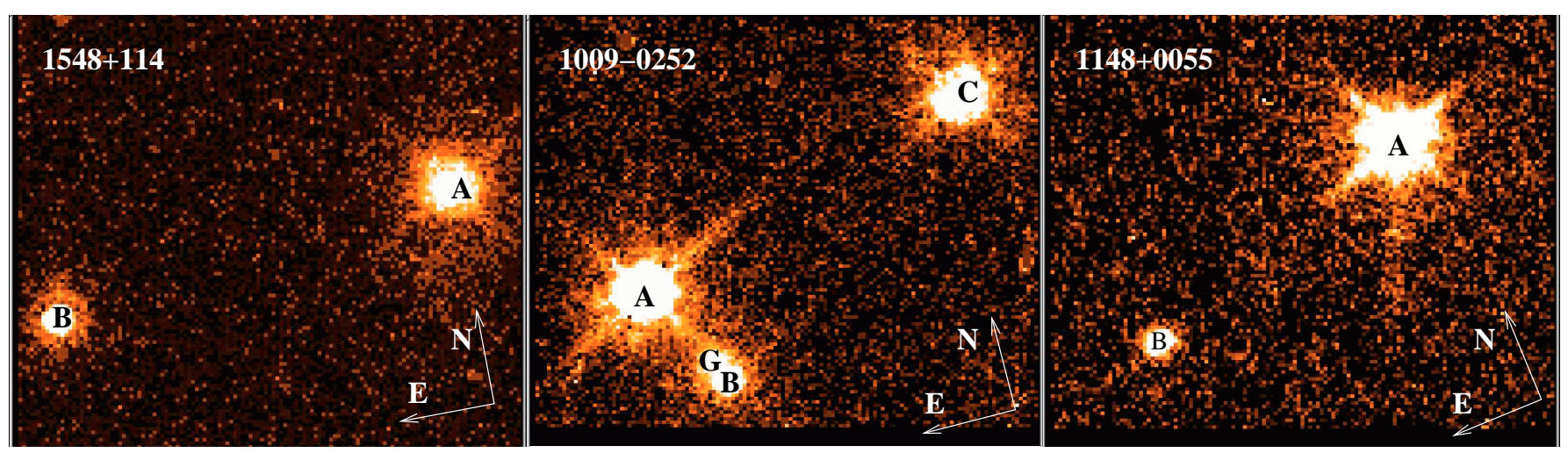

Fig. 1. HST F814W observations of the 3 close quasar pairs with different redshifts known up to-day. In the center, we note the particular case of Q1009-0252 for which the background quasar is lensed by the galaxy G (see Papers I and II for details).

images for about 2900 QSOs: 780 QSOs found in the HST database, 1164 QSOs listed in optical surveys for gravitational lensing (Claeskens 1999), 118 QSOs studied for their environment (Ellingson et al. 1991; Osmer et al. 1998), 281 QSOs observed from the LBQS (Hewett, private communication), 240 quasars observed from the Cerro Tololo Surveys (Maza et al. 1993, 1995, 1996) by Schechter et al. (private communication) and about 300 QSOs from the FBQS survey (Schechter, private communication).

It is of course impossible to list all existing QSO observations present in all observatory archives, but it is reasonable to assume that, on the basis of these 2883 confirmed observations and the numerous applications based on QSO images and spectra (polarization, galactic environment, absorption line correlation, damped Ly-alpha systems,...), about 5000 QSOs have been imaged by the year 2000 with an angular resolution good enough to distinguish companions separated by more than $1 "$. These 5000 QSOs represent $48 \%$ of the 10437 QSOs left in the VV2000 catalog after the 2776 objects identified on objective prism plates have been removed (when they are not reobserved, these objects are strongly biased against close pair detections (Iovino et al. 1996)). For simplicity, we shall assume that $50 \%$ of the remaining QSOs have indeed been re-observed, i.e. our final catalog contains 5218 objects randomly chosen among 10437 QSOs.

The quasars reported in VV2000 can be divided into 4 categories: quasars with a Johnson $B$ magnitude (3864 objects; hereafter population PB), the ones observed in Johnson $V$ (1390 objects; hereafter population $\mathrm{PV}$ ), those in Johnson $R$ (1142 objects; hereafter population PR) and those detected on a Kodak 103-aO emulsion (hereafter $\mathrm{O}$ filter; nearly $B$ filter except in the UV part, see http://aps.umn.edu/datadir/response.html for the response curve; 3841 objects; hereafter population PO). Finally, 26 objects reported with an infrared magnitude but without filter information were removed as well as 174 objects without reported magnitude.

\subsection{Surface density}

Approximately half of the quasars (i.e. populations PB and PV) have a reported $B$ magnitude. Because of this, we have decided to use the cumulative surface density in the $B$ filter. The one derived by Hartwick \& Schade (1990), based on a compilation of different quasar surveys presents numerous advantages. It has been determined up to $B=22.5$ and a correction for incompleteness has been taken into account, including a precise correction for the different observational biases. Furthermore, the surface density has been estimated on the basis of a quite large quasar sample (around 1300 quasars). The curve $\Gamma=b^{(B-a)}$ (where $\Gamma$ is the surface density of quasars brighter than magnitude $B$ ) has been adjusted on the Hartwick and Schade results (i.e. finding respectively for the $b$ and $a$ coefficients 6.91 and 18.47 for $B<19.97$ and 2.26 and 16.41 for $B>19.97$ ). The resulting surface density of quasars has then been used in the calculation of the expected number of pairs (see Sect. 4 for details).

\subsection{The color transformation}

As shown in the previous section, it is necessary to derive a $B$ magnitude for each quasar in the sample. For this purpose, we have calculated the $B-O, B-R$ and $B-V$ color transformations by applying the transmission curve of the different filters to an average spectrum of quasar built from the composite spectra of Zheng et al. (1997) and Francis et al. (1991) (see Sect. 2.3 of Royer et al. 2000 for details). The existence in VV2000 of an observed $B-V$ color allowed us to empirically choose the most appropriate absorption model (i.e. the so-called $B$ model in Royer et al. 2000) and to check the validity (in a particular case) of the synthetic color transformation. It is obvious that this spectrum is representative of an average quasar and that individual spectra may deviate from this one. Nevertheless, as we may see for the $B-V$ subsample in Fig. 2, the theoretical curve deviates from the mean observed $(B-V)(z)$ by less than $0.2 \mathrm{mag}$. On the other hand, we see in Fig. 2 that the $B-R$ transformation gets very important for high values of the redshift (typically $z>4$ ). At redshifts $z \geq 2.5$, the absorbed $\mathrm{Ly}_{\alpha}$ forest appears in the $B$ filter, and both the $B-V$ and $B-R$ color indices increase rapidly. Because the exact amount of absorption is line-of-sight dependent, we have arbitrarily fixed the $B-R$ index to its value at $z=4$ for any redshift larger than 4 . This procedure avoids overestimations of 


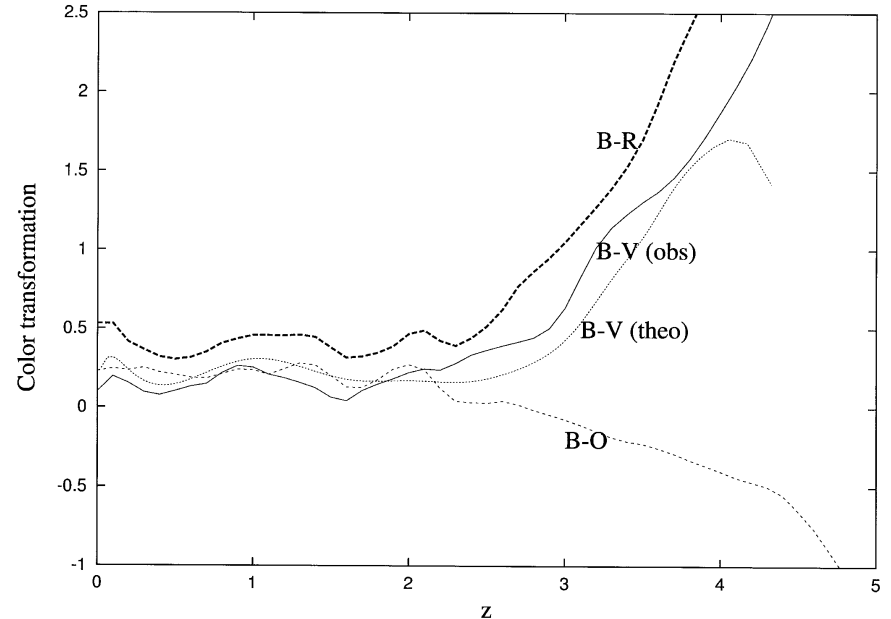

Fig. 2. Color transformations calculated from the transmission curves of the relevant filters applied to a synthetic spectrum of an average quasar (Royer et al. 2000). The $B-V$ solid and dotted lines compare corrections based on observations (see VV2000) with those based on models.

the $B$ mag, and consequently, of the expected number of QSO pairs (see Eq. (1) below).

\section{Expected number of pairs and probability}

As previously suggested by Burbidge et al. (1974), we may apply a very simple a priori method to predict the number of close quasar pairs arising from random projections in a quasar sample. The number of quasars brighter than a limiting magnitude $m$ and lying within $\theta$ arcsec from an arbitrary center is given by:

$<N_{\mathrm{p}}>=2.42 \times 10^{-7} \theta^{2} \Gamma(<m)$,

where $\Gamma(<m)$ represents the number of quasars per square degree brighter than the magnitude $m$. If the center coincides with a quasar of magnitude $m$, this formula fixes the expected number of pairs with a separation smaller than $\theta$ arcsec and a companion brighter than $m$. Each search for a quasar in an area of 5 arcsec radius centered on the target quasar constitutes a trial. Of course, the detection of a nearby quasar is counted as a success. If we apply this technique to $N$ target quasars, we may evaluate the expected number of successes by the relation:

$<N_{\mathrm{p}}>_{\text {tot }}=N<N_{\mathrm{p}}>=2.42 \times 10^{-7} N \theta^{2} \Gamma(<m)$.

The associated probability of finding $k$ pairs in $N$ trials is given by the Poisson statistics:

$P\left(k ;<N_{\mathrm{p}}>_{\text {tot }}\right)=\frac{\left(<N_{\mathrm{p}}>_{\text {tot }}\right)^{k} \mathrm{e}^{-<N_{\mathrm{p}}>_{\text {tot }}}}{k !}$.

\subsection{Results}

First, a correction for the Milky-Way extinction (see Schlegel et al. 1998) has been applied to all quasars in VV2000, as a function of their galactic latitude (typical extinction values are smaller than $0.3 \mathrm{mag}$ ). Following this, the $\mathrm{O}$ (resp. R) magnitudes of the PO (resp. PR) subsamples have been transformed into $B$ magnitudes using the associated synthetic colour transformations (see Sect. 3.2). Subsequently, we have calculated the number of pairs (which is typically of the order of $10^{-4}$ ) expected within an annulus whose inner radius is $1^{\prime \prime}$ and its outer radius $5^{\prime \prime}$, centered on each quasar (see Eq. (1)). Then, we randomly selected subsamples containing half the number of quasars in the catalogue (see Sect. 3) and summed the individual numbers of pairs according to Eq. (2) in order to obtain the total expected number of pairs. After performing $1000 \mathrm{such}$ random selections, we derived the mean total number of pairs in the sub-catalog together with its standard deviation. This has been carried out under various reasonable hypotheses.

Under the most conservative hypothesis (H1), we consider that the faintest object detectable in the field is the target quasar of the pair (let us refer to it as $m_{\text {ref }}$ ). This is probably an overpessimistic hypothesis. Indeed, it is reasonable to assume that, in general, the existing direct images go (at least) one magnitude fainter than $m_{\text {ref }}$ : this constitutes our second hypothesis $(\mathrm{H} 2)$. A third one (H3) consists in assuming that for quasars with a magnitude brighter than 20, all fields reach a depth down to the 20th magnitude. This means that under H3, we consider $\Gamma(m)=\Gamma(20)$ for $m<20$. The last hypothesis (H4) consists in combining $\mathrm{H} 2$ and $\mathrm{H} 3$ : it is equivalent to $\mathrm{H} 2$ if $m>20$ and equivalent to $\mathrm{H} 3$ if $m<20$.

Table 2 shows that under the $\mathrm{H} 1$ hypothesis, the probability of discovering three pairs of quasars with different redshifts is not higher than $0.3 \%$. This value increases to $2.8-12.8 \%$ under the other more realistic hypotheses.

Equation (2) shows that the expected number of pairs is strongly dependent on the outer radius of the annulus within which we are looking for the presence of a companion. Because the $5^{\prime \prime}$ outer radius is a somewhat historical but arbitrary choice, we have also investigated what is happening when we increase the maximum search radius by step of $1^{\prime \prime}$ up to $10^{\prime \prime}$ (i.e. for radius of $\left.6^{\prime \prime}, \ldots, 10^{\prime \prime}\right)$. We find in VV2000 three new discordant redshift pairs with separations of respectively 5.38", $8.97^{\prime \prime}$ and 9.93". Figure 3 shows that for each hypothesis, we do not find a radical change for the probability of discovering the observed pairs: the discovery of the known pairs is marginally unlikely for $\mathrm{H} 1$ but likely given any other realistic hypothesis. As a numerical illustration, under $\mathrm{H} 4$, the probability of discovery of the observed pairs remains between 11 and $20 \%$ within the $5-10^{\prime \prime}$ maximum outer radius.

\subsection{Sources of uncertainties}

Three main sources of uncertainties are clearly identified: photometric errors, color transformations and the adopted surface density of quasars. First we considered random photometric errors up to 0.5 mag for the whole population of quasars. This induces an overestimation of $1-2 \%$ in the expected number of quasar pairs. On the other hand, a systematic error in the color transformations may induce an overestimate (or an underestimate) in the quasar population at a given magnitude and, consequently, a bias in the calculation of the expected number of pairs. This error only affects the PR (1142 objects) and PO (3841 objects) populations and is probably small for 
Table 2. Expected number of QSO pairs with uncertainties (lines 1,2) and probability of observing 0 to 9 pairs (lines 3-12) among 5218 QSOs randomly chosen in VV2000, and under different hypotheses H1-H4 (see text).

\begin{tabular}{l|c|c|c|c}
\hline \hline$N_{\text {pair }}$ & $P(H 1)$ & $P(H 2)$ & $P(H 3)$ & $P(H 4)$ \\
\hline$N_{\text {pair }}$ (tot) & 0.28 & 0.70 & 1.24 & 1.52 \\
$\sigma$ & 0.011 & 0.015 & 0.010 & 0.023 \\
\hline 0 & 0.753 & 0.499 & 0.289 & 0.219 \\
1 & 0.214 & 0.347 & 0.359 & 0.333 \\
2 & 0.030 & 0.121 & 0.223 & 0.253 \\
$\mathbf{3}$ & $\mathbf{0 . 0 0 3}$ & $\mathbf{0 . 0 2 8}$ & $\mathbf{0 . 0 9 2}$ & $\mathbf{0 . 1 2 8}$ \\
4 & $2 \times 10^{-4}$ & 0.005 & 0.029 & 0.049 \\
5 & $1 \times 10^{-5}$ & $7 \times 10^{-4}$ & 0.007 & 0.015 \\
6 & $6 \times 10^{-7}$ & $8 \times 10^{-5}$ & 0.002 & 0.004 \\
7 & $2 \times 10^{-8}$ & $8 \times 10^{-6}$ & $3 \times 10^{-4}$ & $1 \times 10^{-4}$ \\
8 & $8 \times 10^{-10}$ & $7 \times 10^{-7}$ & $4 \times 10^{-5}$ & $2 \times 10^{-4}$ \\
9 & $3 \times 10^{-11}$ & $5 \times 10^{-8}$ & $6 \times 10^{-6}$ & $3 \times 10^{-5}$ \\
\hline
\end{tabular}

the $\mathrm{O}$ population because this filter is close to the $B$ filter (see e.g. Evans 1989), which implies that the $B-O$ correction only varies between 0.1 and 0.3 mag for $z \leq 2$ as we can see from Fig. 2. If we consider that the whole PO population is affected by a systematic error of up to $\pm 0.3 \mathrm{mag}$, and that the PR population is affected by an error of up to $\pm 0.5 \mathrm{mag}$, our simulations indicate that this may induce variations in the expected number of pairs by $25 \%$ under $\mathrm{H} 1$ and by less than $15 \%$ under the other hypotheses. The last main source of uncertainty comes from the surface density of quasars. This error is quantitatively difficult to evaluate, but roughly, we know that it will not change the order of magnitude of our estimates. Indeed, as we can see from Eq. (2), the expected number of pairs is directly proportional to the surface density of quasars. This means that a global overestimate of the surface density by $15 \%$ (which is the typical one sigma error bar on the cumulative surface density) would also induce an overestimate in the number of pairs by $15 \%$.

\subsection{Discussion}

Under various reasonable assumptions, we have derived the corresponding expected numbers of close quasar pairs with different redshifts in VV2000. Our results are found to be compatible with the observations. Moreover, according to Sect. 4.2 we do not expect the uncertainty in these results to exceed $20 \%$. This means that given $\mathrm{H} 3$ or $\mathrm{H} 4$, the expected number of pairs is found, under realistic conditions, to be around 1.5, which is nearly 2 times larger than what was expected in BHS97. This factor 2 comes from the fact that BHS97 derived their expected number of pairs from the small subsamples among which each system has been discovered, and not from a larger sample of quasars observed with a good angular resolution, as we did using random selections of the VV2000 catalogue. On the other hand, since we are left with 3 confirmed QSO pairs instead of the 4 proposed by BHS97, the likelihood to find these QSO pairs in VV2000 is found to be $\sim 10$ to 40 times larger than that computed by BHS97 in their smaller subsamples.

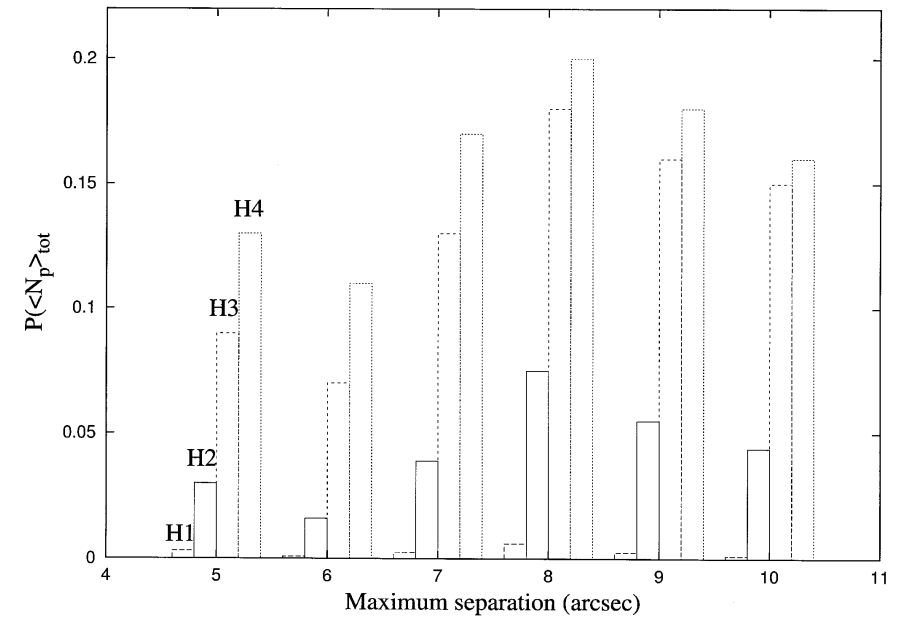

Fig. 3. Probability of discovering the observed number of pairs in VV2000 for 6 different values of the maximum outer search radius.

\section{Conclusions}

Three quasar pairs separated by less than $5^{\prime \prime}$ and with different redshifts $(\Delta z>0.1)$ are identified in the VV2000 catalogue of quasars. Using a simple a priori method, we have determined the expected number of pairs of quasars which should be found in that catalogue. Considering that about 5000 quasars in VV2000 have been imaged in such a way that a companion as close as $1^{\prime \prime}$ would have been discovered and identified, we have calculated using the most conservative hypothesis a probability of $0.3 \%$ of finding the three observed pairs. This result is similar to the most optimistic estimation made in BHS97. Nevertheless, we have shown that under realistic observing conditions, the number of pairs is expected to be between 0.7 and 1.52 , and is consistent with the observations since the associated probability of discovering the three known systems is then between $2.8 \%$ and $12.8 \%$.

On the other hand, we have imaged these pairs with HST and shown the absence of any secondary lensed image in the vicinity of the foreground quasar (see Fig. 1). A precise modeling of the different systems has enabled us to show that for two systems (i.e. Q1009-0252 and Q1148+0055), the amplification induced by the foreground quasar and its environment is very small (typically smaller than 10\%). For the third one (i.e. Q1548+114), we found an amplification factor between 1.06 and 1.72. This clearly indicates that the amplification of the background population of quasars due to foreground ones is probably not at all as high (i.e. $1 \mathrm{mag}$ ) as previously estimated in BHS97.

Finally, if we consider that the model of BHS97 gives a good rough description of the gravitational lensing amplification bias, we have shown that in the extreme case of a background amplification by $0.5 \mathrm{mag}$, the expected number of pairs is doubled, which lets the probability to find three pairs between $2 \%$ and $18 \%$. In the near future, the discovery of additional close pairs of quasars with different redshifts in deeper quasar samples (cf. SDSS, $2 \mathrm{dF}, \ldots$ ) will probably enable us to evaluate more precisely the importance of the amplification bias and to work with statistical results not based on rare events. 
If many pairs are discovered in the future, we may use the statistics as a tool to investigate quantitatively the mean amplification bias.

Acknowledgements. D.S. wants to thank Dr Pierre Royer for the computation of the color transformations and for interesting discussions and Dr D. Schlegel for the availability of his software enabling us to calculate the galactic extinction. We also want to thanks P. Hewett, N. Morgan, P. Schechter, D. Crampton, S. Cristiani, R. Green, R. Kron, G. Richards, D. Schade, R. Webster and H. K. C. Yee who responded to our inquiry on the number of re-observed quasars. We also thank the referee Dr P. Véron, for his constructive comments. Our research was supported in part by PRODEX (Gravitational lens studies with HST), by contract IUAP P5/36 "Pôle d'Attraction Interuniversitaire" (OSTC, Belgium) and by the "Fonds National de la Recherche Scientifique" (Belgium).

\section{References}

Burbidge, G. R., Hoyle, F., \& Schneider, P. 1997, A\&A, 320, 8 (BHS97)

Burbidge, E. M., Beaver, E. A., Cohen, R. D., Junkkarinen, V. T., \& Lyons, R. W. 1996, AJ, 112, 2533

Burbidge, E. M., Burbidge, G. R., \& O’Dell, S. L. 1974, Nature, 248, 568

Claeskens, J.-F., \& Surdej, J. 2002, A\&A Rev., 10, 263

Claeskens, J.-F., Khmil, S. V., Lee, D. W., Sluse, D., \& Surdej, J. 2001, A\&A, 367, 748 (Paper II)

Claeskens, J.-F., Lee, D. W., Remy, M., Sluse, D., \& Surdej, J. 2000, A\&A, 356, 840 (Paper I)
Claeskens, J.-F. 1999, Ph.D. Thesis Soc. Roy. Sci. Liège, 68, 1

Ellingson, E., Yee, H. K. C., Green, R. F. 1991, ApJS, 76, 455

Evans, D. W. 1989, A\&A, 78, 249

Francis, P. J., Hewett, P. C., Foltz, C. B., et al. 1991, ApJ, 373, 465

Hartwick, F. D. A., \& Schade, D. 1990, ARA\&A, 28, 437

Iovino, A., \& Shaver, P. A. 1986, A\&A, 166, 119

Iovino, A., Clowes, R., \& Shaver, P. 1996, A\&AS, 119, 265

Maza, J., Wischnjewsky, M., \& Antezana, R. 1996, RMxAA, 32, 35M

Maza, J., Wischnjewsky, M., Antezana, R., \& Gonzalez, L. E. 1995, RMxAA, 31, 119M

Maza, J., Ortiz, P. F., Wischnjewsky, M., Antezana, R., \& Gonzalez, L. E. 1995, RMxAA, 31, 159M

Maza, J., Ruiz, M. T., Gonzalez, L. E., Wischnjewsky, M., \& Antezana, R. 1993, RMxAA, 25, 51M

Osmer, P. S., Kennefick, J. D., Hall, P. B., \& Green, R. F. 1998, ApJS, 119, 189

Royer, P., Manfroid, J., Gosset, E., \& Vreux, J.-M. 2000, A\&AS, 145, 361

Spinrad, H., \& Smith, H. E. 1975, ApJ, 201, 275

Schechter, P. L., Gregg, M. D., Becker, R. H., Helfand, D. J., \& White, R. L. 1998, AJ, 115, 1371

Schlegel, D. J., Finkbeiner, D. P., \& Davis, M. 1998, ApJ, 500, 525

Urry, C. M., Scarpa, R., O’Dowd, M., et al. 2000, ApJ, 532, 740

Véron-Cetty, M. P., \& Véron, P. 2000, ESO Sci. Rep., 19, 1 (VV2000)

Wampler, E. J. 1997, ApJ, 476, L55

Wampler, E. J., Baldwin, J. A., Burke, W. L., Robinson, L. B., \& Hazard, C. 1973, Nature, 246, 203

Zheng, W., Kriss, G. A., Telfer, R. C., Grimes, J. P., \& Davidsen, A. F. 1997, ApJ, 475, 469 (Erratum: 1998, ApJ, 492, 855) 